\title{
Comparison of simulated and measured field dependent charge injection in mineral oil under de bias
}

\author{
Olof Hjortstam, Christian Sonehag and Joachim Schiessling \\ Power Technologies, ABB Corporate Research \\ SE-721 78, Västerås, Sweden
}

\begin{abstract}
The present study aims at the understanding of charge injection from metal electrodes into mineral oil under DC stress. The work focuses on the field dependence of the charge injection. In a previous article the electrical field in a blank oil gap with parallel electrodes is measured with a so called Kerr electro-optic technique. The data show that the electrical field is close to symmetric for low fields $(\sim 0.1 \mathrm{kV} / \mathrm{mm})$ but strongly asymmetric at higher fields $(\sim 1 \mathrm{kV} / \mathrm{mm})$. In the present study an ion drift model is used to analyze the data. We show that an ion drift model including a field dependent injection term can reproduce the measured results fairly well. Furthermore, the comparison of measured and simulated results shows that the charge injection is stronger from an electrode with positive polarity compared to one of negative polarity.
\end{abstract}

\section{Introduction}

During the last years it has been a push for Ultra High Voltage Direct Current (UHVDC) transmission systems, due to the rising demands on transmitting electrical power over long distances. A key component in such systems is the mineral oil insulated HVDC converter transformer. The increased voltage levels are calling for a deeper understanding of high field phenomena under $\mathrm{dc}$ stress in mineral oil based insulation systems.

It is a complex issue to predict the electrical fields in mineral oil insulation systems. From the literature it is clear that the ionic transports in the insulation liquids have to be taken into account in order make good predictions of the electrical field distribution under voltage stress. The so called ion-drift models have been discussed by several authors during the past decades [1-3]. Such models include dissociation of ions from pre-existing ion pairs as well as transport of ions in electrical field. Models for injections from metallic contacts are also discussed in the literature [4].

In Ref. [2] Kerr measurements of the electrical field over a $19 \mathrm{~mm}$ mineral oil gap are presented. At $2 \mathrm{kV}$ a symmetric parabolic distribution of the electrical field along the oil gap is observed. At $20 \mathrm{kV}$ an asymmetric field distribution is observed. In Ref [2] the asymmetries were explained by assuming that the positive and negative electrical ionic mobility differ significantly. However, resent measurements, on mineral oil, shows no difference of the measured ionic mobility for the two polarities [5]. In the current article we investigate how a field dependent injection from metal contacts can be used to understand how an increased voltage can change the electrical field from a symmetric to an asymmetric field distribution.

\section{Experimental data}

The Kerr technique uses the so called electro-optical Kerr effect to measure the electrical field in the oil. A laser beam is aligned through the oil and the difference in the polarization angle is used as a measure of the electrical field. In the present article earlier measurements [2] of the electrical field in mineral oil is compared to new simulations. The measurement data is taken from an experimental geometry with a $19 \mathrm{~mm}$ long gap and parallel electrodes. The gap is filled with mineral oil. One dimensional data of the electrical fields along the $19 \mathrm{~mm}$ oil gap is extracted from Ref [2] and is compared to new results from the simulation model used in the present work.

\section{The ion drift model}

The model used in this article is similar to that described in $[2,3]$ and it is outlined below. The ion drift model described below is implemented and solved in the simulation finite-element software Comsol Multiphysics V4.3 [6]. The simulations are done in a 1-dimensional geometry with a $19 \mathrm{~mm}$ wide oil gap.

\subsection{The governing equations}

In the model, transformer oil is considered as a weak electrolyte filling a gap between two plane metallic electrodes. At thermodynamic equilibrium (at zero field limit), the concentrations of positive $p$ and negative $n$ ions in the oil bulk are equal and are much less than the density of neutral molecules $c$. The equilibrium ion concentrations, $\mathrm{n}_{0}$ and $\mathrm{p}_{0}$ are defined by the ohmic conductivity $\sigma$ :

$$
p_{0}=n_{0}=\sigma / q\left(\mu_{p}+\mu_{n}\right)
$$

Here, $q$ is the elementary charge; $\mu_{p}$ and $\mu_{n}$ are the electrical mobilities of positive and negative ions; the subscript " 0 " indicates equilibrium densities. The ions 
are continuously being generated from the dissociation of ionic pairs and, conversely, ionic pairs are formed by the recombination of ions. The process is described by the rate equation

$$
d p / d t=d n / d t=k_{D} c-k_{R} p n
$$

where $k_{D}$ and $k_{R}$ are the dissociation and recombination constants, respectively. The recombination constant is found using Langevin approximation

$$
k_{R}=q\left(\mu_{p}+\mu_{n}\right) / \varepsilon_{0} \varepsilon_{r}
$$

Here, $\varepsilon_{0}$ and $\varepsilon_{r}$ stand for the permittivity of vacuum and the dielectric constant of oil, respectively.

The dissociation constant, $\mathrm{k}_{\mathrm{D}}$, will depend on the electric field according to Onsager theory [7]. Here the applied electric field will lower the potential barrier binding negative and positive ions into ion pairs.

$$
\begin{aligned}
& k_{D}=k_{D}^{0} F(E)=k_{D}^{0} \frac{I_{1}(4 b)}{2 b} \\
& b=\sqrt{\frac{q^{3} E}{16 \pi \varepsilon_{0} \varepsilon_{r} k^{2} T^{2}}}
\end{aligned}
$$

where $I_{1}$ is the modified Bessel function of first order, E the electric field strength, k Boltzmann's constant and T the absolute temperature.

From equation (2) assuming thermodynamic equilibrium we get that

$$
k_{D}^{0}=k_{R} \frac{n_{0}^{2}}{c}
$$

If an external electric field $E$ is applied, charge carriers in the bulk of the fluid are separated due to electrostatic forces and experience drift and diffusion. Their drift velocities are defined as

$$
\vec{w}_{p, n}=\mu_{p, n} \vec{E}
$$

while the magnitudes of the diffusive fluxes are proportional to the gradients of the ions' densities. The diffusion coefficients $D_{p, n}$ can be found using Einstein's relation:

$$
D_{p, n}=\mu_{p, n} \cdot k T / q
$$

Thus, equation (2) can be extended to the system of equations for time and space variations of the ion densities:

$$
\begin{aligned}
& \partial p / \partial t+\nabla \cdot\left(\mu_{p} \vec{E} p-D_{p} \nabla p\right)=S \\
& \partial n / \partial t-\nabla \cdot\left(\mu_{n} \vec{E} n+D_{n} \nabla n\right)=S \\
& S=k_{D} c-k_{R} p n
\end{aligned}
$$

Equations (8) contain field dependent coefficients and, hence, they should be supplemented by Poisson's equation for the electric potential, $\phi$. This allows for obtaining electric field distributions in the liquid affected by the space charge:

$$
\nabla \cdot\left(\varepsilon_{0} \varepsilon_{r} \nabla \phi\right)=-q(p-n), \quad \vec{E}=-\nabla \phi
$$

\subsection{Boundary conditions}

Electrical boundary conditions for (9) are set to the chosen potential at the metal electrodes. All other external boundaries are set to Neumann type.

The physical processes on metal-oil interfaces have to be obeyed and the continuity of the electric current preserved. Therefore, conditions on the surfaces of the metallic electrodes in contact with oil, are to be specified. There are two types of boundary conditions, inflow and outflow, which need to be provided for ionic fluxes.

In the work by Alj et al. [4] the injection of ions was described as a three step process. 1) A fraction of the ion pairs in the liquid are attached to the contact. 2) Charge is transferred between the metal and ion pairs, leading to dissociation of the ion pairs. One polarity of the ions is neutralized while the other ion polarity remains charged. 3) The remaining ions can be extracted from the interface by the electrical field. This model implies that the injected ions are identical to the ion species created by dissociation in the bulk. Taking the image charge into account an expression for the ion concentrations at the boundaries are derived [4]:

$$
\begin{aligned}
& p_{i n j}=A_{p} p_{0} F_{i}(E)=A_{p} p_{0} \frac{1}{2 b K_{1}(2 b)} \\
& n_{i n j}=A_{n} n_{0} F_{i}(E)=A_{n} n_{0} \frac{1}{2 b K_{1}(2 b)}
\end{aligned}
$$

where $K_{1}$ is the modified Bessel function of the second kind and order one. $A_{n}$ and $A_{p}$ are injection strength parameters used in the present work to tune the strength of the ionic injection, for each polarity. In the original work [4] these parameters were identical to one. In the present study, the injecting metal surfaces are defined as a boundary supplying a flow of ions into oil that is given by the concentration (equation 10) times the ionic speed $\left(\mu_{n} E\right.$ or $\left.\mu_{p} E\right)$. The parameters $A_{p}$ and $A_{n}$ will be used for tuning the injection strength in order to match experimental data. If one of the injection parameters is set to zero the injection will be unipolar whereas if both injection parameters are non zero the injection will be bipolar. 
For the outflow of ions, non-blocking properties of the contacts are assumed. All ions that reach the electrode are assumed to be able to exit the domain through the boundary.

In the simulations presented below the ionic mobility $\mu_{\mathrm{n}}$ and $\mu_{\mathrm{p}}$ are both set to $10^{-9} \mathrm{~m}^{2} / \mathrm{Vs}$. The equilibrium ionic densities $\mathrm{n}_{0}$ and $\mathrm{p}_{0}$ are set to values giving an equilibrium conductivity of $0.5 \cdot 10^{-12} 1 / \Omega \mathrm{m}$. This value mach the measured oil conductivity of the studied mineral oil [2].

\section{Comparison of simulated and measured results}

In figure 1 and 2 measured as well as simulated electrical steady state fields in the $19 \mathrm{~mm}$ wide oil gap are shown for applied fields of $2 \mathrm{kV}$ and $20 \mathrm{KV}$ respectively. The measured data is taken from Ref. [2]. Let us first focus on the experimental findings. At $2 \mathrm{kV}$ (figure 1) we notice that the electrical field, as a function of position in the oil gap, follows a parabolic shape. In Ref. [2] this behavior is explained by an ion drift model without any injection. The sweep out of ions that are generated by ion pair dissociation in the oil gives rise to hetero-charges around the contacts. These hetero-charges increase the field close to the contacts and give a field minimum in the middle of the gap. Looking at the measured field at $20 \mathrm{kV}$ (figure 2) a different type of field distribution is found. The field is constantly increasing going from the positive electrode to the negative electrode. This type of field distribution can't be reproduced by an ion-drift model without injection and with equal ionic mobility for both ion polarities. In Ref. [2] it was argued that the unsymmetrical field distribution was caused by a different ionic mobility for positive and negative ions. However, more resent results show that such difference in the ionic mobility does not exist [5]. In the present study we are investigating if a field dependent injection could explain this asymmetric field distribution.

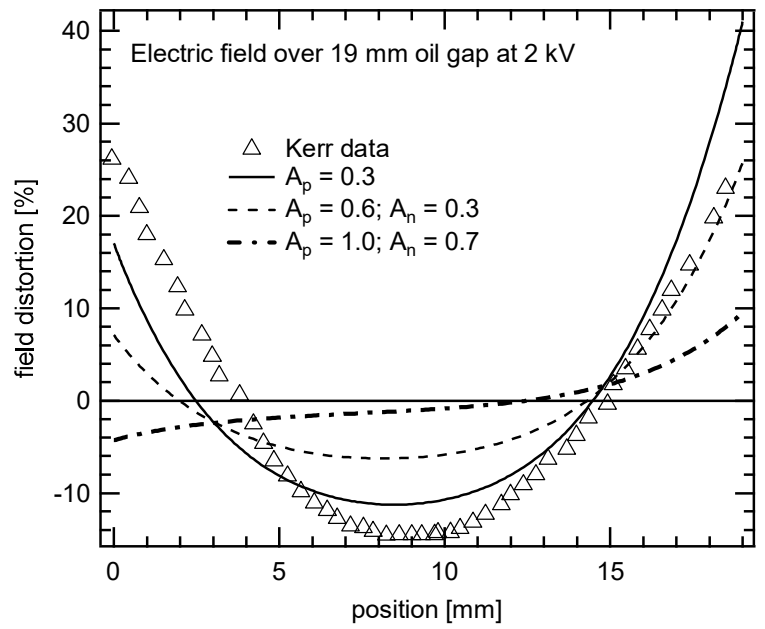

Figure 1 Measured and simulated field distribution over the $19 \mathrm{~mm}$ oil gap at $2 \mathrm{kV}$.

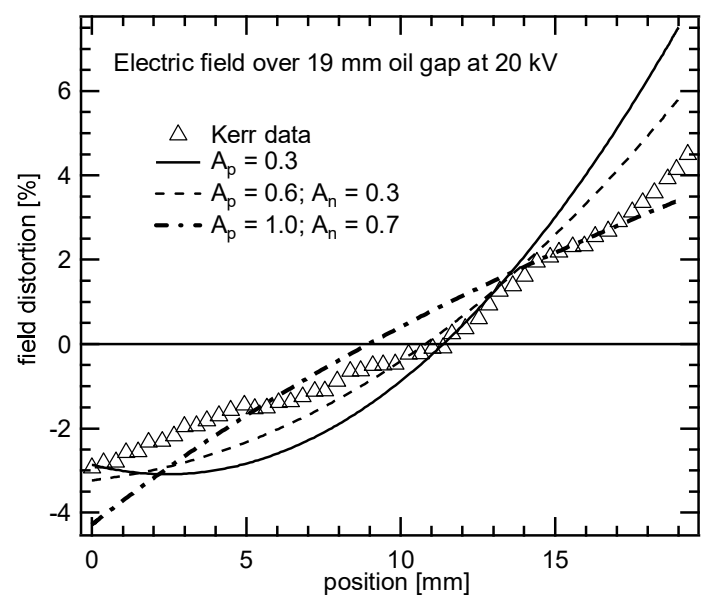

Figure 2 Measured and simulated field distribution over the $19 \mathrm{~mm}$ oil gap at $20 \mathrm{kV}$.

In order to systematically study how the field dependent injection affects the field distortion we started to investigate the unipolar case. Different values (in the range of 0 to 1.0) of $A_{p}$ was tested whereas $A_{n}$ was kept to zero. A general observation is that larger injection strength gives a larger asymmetry in the field distortion. The injection of positive ions reduces the field next to the positive electrode due to the homo charge that is built up. The best match to the measured data at $2 \mathrm{KV}$ occurs at a low (or zero) injection strength and the best fitting for $20 \mathrm{kV}$ occurs for values of $A_{p}$ above 0.5 . The value of $A_{p}$ that gives the best trade of between the matching of $2 \mathrm{kV}$ and the $20 \mathrm{kV}$ data are found to be $A_{p}=0.3$. The simulated field distortion for positive unipolar injection with strength $A_{p}=0.3$ is shown in figure 1 and 2 .

Next the field distortion at bipolar injection was investigated. If the injection strength was set equal for both positive and negative polarity the field distortions remain symmetric (not shown). Different combinations of $A_{p}$ and $A_{n}$ was tested under the assumption that $A_{p}$ $A_{n}=0.3$ (i.e. keeping the net charging of the oil at a constant level). The results from such simulations are shown in figure 1 and figure 2 . Notice that for $2 \mathrm{kV}$ the best match to the measured data occurs for the unipolar case whereas the best match for $20 \mathrm{kV}$ occurs for $A_{p}=1.0$ and $A_{n}=0.7$. The best overall match for both 2 $\mathrm{kV}$ and $20 \mathrm{kV}$ in the bipolar case is obtained for the parameter settings $A_{p}=0.6$ and $A_{n}=0.3$

From the present analysis it is found that a field dependent injection model can be used to explain the transition from a symmetric (parabolic) distribution of the electrical field through the oil gap at $2 \mathrm{kV}$ to an unsymmetric distribution at $20 \mathrm{kV}$. Furthermore, it is clear that injection of positive ions dominates over the injection of negative ions. However it is not possible to conclude whether the injection is unipolar or bipolar is however not possible. 


\section{Conclusion}

In this paper, a comparison of measured and simulated electrical field in a $19 \mathrm{~mm}$ mineral oil gap is presented. At low voltage $(2 \mathrm{kV})$ the electrical field shows a symmetric parabolic distribution whereas at high voltage $(20 \mathrm{kV})$ the electrical field increases linearly from the positive to the negative electrode. The strong difference in the field distribution can be explained by a field dependent injection that gives a substantially higher injection at the higher voltage. From the analysis we also conclude that the injection from positive electrodes is stronger than from negative electrodes. This is in line with recent findings from current measurements in coaxial geometries [8]. From the existing data it was not possible to determine if the injection is a pure unipolar injection (only positive injection) or a bipolar injection with dominating positive injection $\left(A_{p}>A_{n}\right)$.

\section{References}

[1] A. Denat "Etude de la conduction electrique dans le solvants non polaires", Thesis L'Université Scientific at Médicale et L'Institute Nationale Polytechnique, 1982.

[2] U. Gäfvert, A. Jaksts, C. Törnkvist and L. Walfridsson, "Electrical Field Distribution in Transformer Oil”, IEEE Trans. El. Ins. Vol 27, No 3, june 1992, p647-660.

[3] U. Gäfvert, O.Hjortstam, Y. Serdyuk, C. Törnkvist and L. Walfridsson "Modeling aand Measurements of Electric Fields in Composite Oil/Cellulose Insulation", Ann. Rep. IEEE Conf. Elec. Insul. Diel. Phenom., pp 154-157, 2006.

[4] A. Alj, A. Denat, J.P. Gosse, B.Gosse "Creation of Charge Carriers in Nonpolar Liquids", IEEE Trans. El. Ins. Vol 20, No 2, April 1985.

[5] L. Yang, M.S. Sadeh, J. Schiesling, O.Hjortstam, Y.V. Serdyuk and S.M. Gubanski "Measurements of Ion Mobility in Transformer Oils for HVDC applications", Proc. Int. Conf. High Volt. Eng. Appl., Shanghai, China Sept 2012, p464.

[6] Comsol Multiphysics v4.2, COMSOL AB, www.comsol.com

[7] L. Onsager, "Deviation from Ohm's Law in Weak Electrolytes", Journal of Chemical Physics, Vol. 2, 1934, pp 599-615.

[8] J. Schiessling, D.K. Ramesh, Y.V. Serdyuk and O.Hjortstam, "Measurement techniques for identifying polarity dependence of ion injection in transformer oil", NORDIS Conf. Proceeding, Trondheim, Norway, June 2013. 